\title{
PERFIL ANTROPOMÉTRICO, CARDIOMETABÓLICO E QUALIDADE DE VIDA DE MULHERES DIABÉTICAS MENOPAUSADAS.
}

\author{
Thays da Silva Garrido', Mayane Santos Arantes¹, Vitória De Medeiros Marçal', Mayara Moura \\ Alves da Cruz', Gabriele de Lima Gazani Brito², Sebastião Pereira Neiva Neto³, Francis Lopes \\ Pacagnelli $^{1}$, Gabriela Andrade Piemonte Lopes ${ }^{1}$
}

Universidade do Oeste Paulista - UNOESTE, ${ }^{1}$ Curso de Fisioterapia, ${ }^{2}$ Faculdade de Medicina, ${ }^{3}$ Curso de Química, Pres

e.mail: thayz.sg@hotmail.com

\section{RESUMO}

Com os anos, a mulher sofre alterações progressivas da capacidade de adaptação do organismo e qualidade de vida devida alterações hormonais advindas do climatério e menopausa, evidenciando situações como depressão, sobrepeso, e desenvolvimento de fatores de riscos cardiometabólicos, aumento de doenças crônicas como hipertensão e diabetes mellitus. $O$ estudo objetivou avaliar o perfil antropométrico, cardiometabólico e qualidade de vida de mulheres diabéticas menopausadas. Participaram 12 mulheres com diabetes mellitus tipo 2 e analisados dados antropométricos, cardiometabólicos, a qualidade de vida pelo questionário SF-36 e Questionário da Saúde da Mulher-QSM. A análise revelou mulheres com média de idade de $59,33 \pm 9,67$ anos, IMC de $29,57 \pm 7,03 \mathrm{~kg} / \mathrm{m}^{2}$ e a CA de $111,33 \pm 14,35 \mathrm{~cm}$. Os limites dos aspectos emocionais avaliados pelo SF-36 e a depressão pelo QSM foram os domínios mais acometidos. Os resultados mostraram que o perfil antropométrico e cardiometabólicos da população avaliada, compromete a saúde e a qualidade de vida em mulheres na menopausa.

Palavras-chave: Diabetes Mellitus. Menopausa. Climatério. Qualidade de vida. Antropometria.

\section{ANTHROPOMETRIC, CARDIOMETABOLIC PROFILE AND QUALITY OF LIFE IN MENOPAUSAL DIABETIC WOMEN.}

\begin{abstract}
Over the years, the woman undergoes progressive changes the adaptability of the body and quality of life due to hormonal changes resulting from climacteric and menopause, evidencing situations like depression, overweight, and development of cardiometabolic risk factors, increase in chronic diseases such as hypertension and diabetes mellitus. The study aimed to evaluate the anthropometric, cardiometabolic profile and quality of life in menopausal diabetic women. Participated in12 women with type 2 diabetes mellitus and analyzed anthropometric and cardiometabolic data, the quality of life by SF-36 and Women's Health Questionnaire. The analysis showed women with a mean age of $59.33 \pm 9.67$ years, BMI $29.57 \pm 7,03 \mathrm{~kg} / \mathrm{m}^{2}$ and the CA of $111.33 \pm 14,35 \mathrm{~cm}$. The emotional aspects limities evaluated on SF-36 and the depression on WHQ were the aspects mostly affected. The results showed that the anthropometric and cardiometabolic profile in the population studied, undermines the health and quality of life in women during the menopause period.
\end{abstract}

Keywords: Diabetes Mellitus.Menopause. Climacteric. Quality of life. Anthropometry. 


\section{INTRODUÇÃO}

Nos últimos tempos o antigo sexo frágil vem conquistando mudanças em seu perfil sociocultural, buscando seus direitos na sociedade, disputando espaço no mercado de trabalho, sendo reconhecida e valorizada por seus méritos. Somente a função de dona de casa e mãe deixou de ser o auge de sua existência'. Com o passar dos anos, a mulher sofre alterações fisiológicas, bioquímicas e psicológicas, caracterizados pela perda progressiva da capacidade de adaptação do organismo e qualidade de vida, considerado como período de vulnerabilidade ocorrendo alterações no processo de envelhecimento vivenciando transtornos advindos das alterações hormonais ${ }^{2-4}$.

Com o decorrer dos anos, ocorre a menstruação, o climatério e a menopausa como um fator de diminuição na qualidade de vida ${ }^{1}$. A menstruação é designada o fim do processo maturacional e início do período reprodutivo da mulher, o ciclo natural ocorre em média a cada 28 dias, e duração de 5 dias ${ }^{5}$. O climatério é o período em que ocorre a transição da fase reprodutiva para a fase não reprodutiva, com diversas alterações fisiológicas no organismo que se inicia por volta dos 35 e 40 anos, podendo chegar aos 65 anos $^{6,7}$.

Não existe uma programação determinada para o início da menopausa, porém a média de idade geralmente varia em torno de 45 e 55 anos $^{8}$. Segundo Ferrari “à medida que a ciência se aprofunda no conhecimento das secreções de hormônios por parte do ovário, vai se chegando a compreensão de que a síndrome climatérica obedece, em grande parte a falta de ditas substâncias". A menopausa é caracterizada como o período da última menstruação do ciclo reprodutivo, decorrente da falência esteroídica ovariana?

Muitos sintomas atribuídos à transição menopáusica como a ansiedade e depressão não sofreram modificações. Enquanto outros autores admitem que as alterações hormonais deste período geraram distúrbios emocionais, mesmo em mulheres consideradas sadias do ponto de vista emocional ${ }^{9}$. Nesta fase há um aumento no de ganho de peso, modificações na distribuição da gordura e doenças crônicas ${ }^{10}$. Essa mudança no perfil de risco cardiovascular coincide com o climatério e caracterizam-se pelo surgimento ou piora de alguns fatores ${ }^{11}$.

De acordo com a Pesquisa Nacional por Amostra de Domicílios em 2000, haviam 171 milhões portadores de diabetes no mundo, estima-se que em 2030 o Brasil terá cerca de 11,3 milhões de diabéticos. 0 diabetes mellitus tipo 2 está entre as doenças crônicas este decorrente da resistência insulínica herdada ou adquirida na presença de uma falência das células beta geneticamente determinadas, sendo incapaz 
de efetuar sua função no organismo ${ }^{12-14}$ ou por uma ação insuficiente dos hormônios sendo alterada durante a menopausa ${ }^{15}$.

A qualidade de vida se fundamenta na compreensão das necessidades humanas. A Organização Mundial de Saúde define qualidade de vida como "a percepção do indivíduo de sua posição na vida, no contexto cultural sistemas e valores no qual ele vive em relação aos seus objetivos, expectativas, padrões e preocupações"16. A mulher que antes tinha sua vida ativa se dedicava ao trabalho, família, de repente, passa a vivenciar transtornos com alterações de humor, modificações da libido, alteração na memória, alterando a personalidade, necessitando de um auxílio durante a transição da vida ${ }^{17}$.

Muitos sinais e sintomas resultam da queda do estrogênio circulante, com redução de massa magra e aumento de massa adiposa, sobretudo na região abdominal. A obesidade é caracteriza por um acúmulo de gordura corporal em excesso, passando a comprometer a saúde do indivíduo, estando associada ao aumento dos riscos para doenças cardiovasculares, hipertensão arterial, entre outros ${ }^{18}$. Com isso o objetivo do estudo foi analisar perfil antropométrico, cardiometabólico e qualidade de vida de mulheres diabéticas menopausadas.

\section{MÉTODO}

Este trabalho foi submetido à apreciação do Comitê de Ética em Pesquisa e ao Comitê Assessor de Pesquisa Institucional, aprovado através do número 1974. A pesquisa esteve em consonância com o Conselho nacional de ética e pesquisa sob resolução $n^{\circ}$ 466/2012.

Estudo transversal, de delineamento descritivo, no qual a amostra foi composta por 12 portadoras de diabetes mellitus tipo 2 , no período do climatério ou menopausa, residentes no estado de São Paulo.

As mulheres foram submetidas ao termo de consentimento livre e esclarecido, informando e esclarecendo o assunto da pesquisa.

Foi aplicado o questionário de qualidade de vida Medical Out Comes Study (SF 36) multidimensional formado por 36 itens, 8 domínios. Apresenta um escore final de 0 a 100 (obtido por cálculo do RawScale), onde 0 corresponde ao pior estado geral de saúde e 100 corresponde ao melhor estado de saúde ${ }^{19}$. O Questionário da Saúde da Mulher (Women's Health Questionnaire) (QSM) consta de 37 questões, oferecendo quatro alternativas como possibilidade de resposta dispostos aleatoriamente. As alternativas das questões $7,10,21,25,31$ e 32 aparecem em ordem inversa à ordem das outras questões. Portanto, no cálculo dos escores, essas questões tiveram seus 
resultados transformados, isto é, de 1 para 4; de 2 para 3 ; de 3 para 2 e de 4 para $1^{20}$.

Foram verificados dados antropométricos das pacientes como o IMC através da fórmula: IMC=Peso/Altura ${ }^{2}{ }^{21}$. A medida da circunferência abdominal (CA) realizada entre a crista ilíaca e a última costela $^{21}$, verificação da glicemia, aferição da pressão arterial (PA) com um período de repouso de 3 a 5 minutos antes de verificar ${ }^{22}$.
Os dados foram apresentados de maneira descritiva por média e desvio padrão.

\section{RESULTADOS}

A idade, os dados antropométricos e cardiometabólico são expostos em média e desvio padrão na tabela 1.

Tabela 1. Média e desvio padrão da amostra geral estratificado dos parâmetros antropométricos e cardiometabólicos de mulheres diabéticas tipo 2 no climatério e menopausa.

\begin{tabular}{lc}
\hline Parâmetros & Avaliação $(\mathrm{n}=12)$ \\
\hline Idade (anos) & $59,33 \pm 9,67$ \\
Peso $(\mathrm{Kg})$ & $79,13 \pm 15$ \\
Altura $(\mathrm{cm})$ & $1,54 \pm 0,06$ \\
Índice de Massa Corpórea $\left(\mathrm{kg} / \mathrm{m}^{2}\right)$ & $29,57 \pm 7,03$ \\
Circunferência Abdominal $(\mathrm{cm})$ & $111,33 \pm 14,35$ \\
Glicemia $(\mathrm{mg} /$ dl) & $216,16 \pm 117,91$ \\
Pressão Arterial Sistólica $(\mathrm{mmHg})$ & $121 \pm 7,68$ \\
Pressão Arterial Diastólica $(\mathrm{mmHg})$ & $76,66 \pm 9,84$ \\
Pressão Arterial Media $(\mathrm{mmHg})$ & $91,44 \pm 8,25$
\end{tabular}

Em relação à aplicação do SF-36, os escores gerais de cada subgrupo estão apresentados na tabela 2 através da média e desvio padrão. 
Tabela 2. Média e desvio padrão da amostra geral estratificado: Escore para aplicação do questionário de qualidade de vida Medical Out comes Study (SF 36) de mulheres diabéticas tipo 2 no climatério e menopausa.

\begin{tabular}{lc}
\hline Parâmetros & Avaliação $(n=12)$ \\
\hline Capacidade Funcional & $56,66 \pm 31,64$ \\
Limitação Aspectos Físicos & $52,08 \pm 41,91$ \\
Dor & $54,05 \pm 25,06$ \\
Estado Geral de Saúde & $51,66 \pm 20,48$ \\
Vitalidade & $59,16 \pm 24,19$ \\
Aspectos Sociais & $75 \pm 31,07$ \\
Limitação Aspectos Emocionais & $50,05 \pm 41,75$ \\
Saúde Mental & $68 \pm 22,39$ \\
\hline
\end{tabular}

As médias e desvios padrão dos

escores obtidos pelo QSM estão

apresentados na tabela 3.

Tabela 3. Média e desvio padrão da amostra geral estratificado: Escore gerais e por sintomas obtidos por meio da aplicação do QSM de mulheres diabéticas tipo 2 no climatério e menopausa.

\begin{tabular}{lc}
\hline Parâmetros & Avaliação $(n=12)$ \\
\hline Depressão & $3,16 \pm 0,42$ \\
Sintomas Somáticos & $2,48 \pm 0,60$ \\
Memória/Concentração & $2,80 \pm 1,16$ \\
Sintomas Vasomotores & $2,70 \pm 1,04$ \\
Ansiedade/Tremores & $2,97 \pm 0,76$ \\
Comportamento Sexual & $2,58 \pm 0,89$ \\
Problema de sono & $2,58 \pm 0,94$ \\
Sintomas Menstruais & $2,89 \pm 0,58$ \\
Atratividade & $2,88 \pm 0,88$ \\
\hline
\end{tabular}

\section{DISCUSSÃO}

Os valores do IMC e CA constituem o perfil antropométrico das mulheres avaliadas. De acordo com os resultados, observou-se a média do IMC na categoria "pré-obesidade" preconizado pela OMS, uma vez que os valores estejam entre 25 - 29,99, considerando aumento no risco de comorbidade. Os valores da CA obtidos neste estudo sugere risco "aumentado substancialmente" de doenças associadas à obesidade $^{21}$.

Um fator importante em mulheres na menopausa é que o aumento na prevalência de sobrepeso e obesidade tem se mostrado crescente, esse aumento é ainda maior na menopausa, atingindo, aproximadamente, $60 \%$ das mulheres, provavelmente em 
virtude das alterações metabólicas e à predisposição genética de cada mulher ${ }^{23}$.

A obesidade também está relacionada com o desenvolvimento de diabetes mellitus, sendo aumentado conforme aumenta o $\mathrm{IMC}^{24}$. Este fato pode ser observado no presente estudo, onde a média de glicemia pós-prandial foi de $216,16 \mathrm{mg} / \mathrm{dl}$.

A doença cardiovascular pode ser um importante determinante de morbidade em mulheres a partir da quinta década, estando associada à obesidade, dislipidemia, intolerância à glicose e hipertensão ${ }^{25}$. Porém, em nosso estudo a pressão arterial esteve dentro dos limites considerados normais para indivíduos acima de 18 anos $^{26}$.

Há vários estudos acerca dos efeitos das alterações hormonais devido a menopausa causando sintomas de irritabilidade, ansiedade, acarretando incapacidade e redução na qualidade de vida dessas mulheres ${ }^{27-29}$, sendo de acordo com o presente estudo, onde pôde observar as limitações dos aspectos emocionais, pelo SF36 e a depressão, pelo QSM, como sendo os domínios que mais tiveram acometidos.

\section{CONCLUSÃO}

Os resultados mostraram IMC e CA elevada, aumentando o risco de comorbidade na população avaliada, comprometendo assim, a saúde e a qualidade de vida em mulheres diabéticas na menopausa.

\section{AGRADECIMENTOS E CONFLITO DE INTERESSE \\ Os autores declaram não haver} qualquer potencial conflito de interesse que possa interferir na imparcialidade deste trabalho científico.

\section{REFERÊNCIAS}

1. Teixeira MZ. Distúrbios Climatério e Tratamento Homeopático. Homeopat. Bras. 2002; 8(1): 29-43.

2. Ciolac, EG, Guimarães GV. Exercício físico e síndrome metabólica. RevBrasMed Esporte. 2004;10(4):319.

3. Santos AA, Bertato FT, Montebelo MIL, Guirro ECO. Effect of proprioceptive training among diabetic women. Rev Bras Fisioter. 2008;12(3):183-187. DOI: http://dx.doi.org/10.1590/S141335552008000300005 .

4. Fechine BRA, Trompieri N. O processo de envelhecimento: as principais alterações que acontecem com o idoso com o passar dos anos. Rev Cient Inter. 2012;1(3):632.DOI: http://dx.doi.org/10.6020/1679$\underline{9844 / 2007}$

5. Peixoto LN, Pachioni FSM, Pelai EB, Mantovani AM, Pinto MC, Palma MR, et al. Terapia de consciência corporal em mulheres pós-cirurgia de câncer de mama, consciência corporal e câncer de mama.2014. 
6. Castilho SD, Pinheiro CD, Bento CA, BarrosFilho AA, Cocetti $M$. Tendência secular da idade da menarca avaliada em relação ao índice de massa corporal. Arq Bras Endocrinol Metab. 2012;56(3):195-200. DOI:http://dx.doi.org/10.1590/S000427302012000300008

7. Freitas KM, Silva AR, Silva RM. Mulheres vivenciando o climatério. Acta Scientiarum Health Sciences.2004;26(1):121-128.

8. Rocha MDHA, Rocha PA. Do climatério à menopausa. Revista científica do itpac Faculdade de Ciências Humanas Econômicas e da Saúde de Araguaína. 2010;3(1):24-27.

9. Neto JAF, Figuerêdo ED, Barbosa JB, Barbosa FF, Costa GRC, Nina VJS, et al. Metabolic Syndrome and Menopause: CrossSectional Study in Gynecology Clinic. Arq Bras Cardiol.2010;95(3):339-345.

10. Toscano CM. As campanhas nacionais para detecção das doenças crônicas nãotransmissíveis: diabetes e hipertensão arterial. Cien Saud Col. 2004;(4):885-895.

11. Monteiro LZ, Fiani CRV, Freitas MCF, Zanetti ML, Foss MC. Decrease in Blood Pressure, Body Mass Index and Glycemia after Aerobic Training in Elderly Women with Type. 2010;95(5):563-570.

12. Manna TD, Damiani D, Setian N. Síndrome Metabólica: revisão. 2006;28(4):272-277.

13. Francisco PMSB, Belon AP, Barros MBA, Carandina L, Alves MCGP, Goldbaum M, et al. Diabetes auto-referido em idosos: prevalência, fatores associados e práticas de controle. Cad. Saúde Pública. 2010;26(1):175184. DOI: http://dx.doi.org/10.1590/S0102311X2010000100018.
14. Souza JR, Oliveira RT, Blotta MH, Coelho OR. Serum Levels of Interleukin-6 (IL-6), Interleukin-18 (IL-18) and C-Reactive Protein (CRP) in Patients with Type-2 Diabetes and Acute Coronary Syndrome without STSegment Elevation.

ArqBrasCardiol.2008;90(2):86-90.

DOI:http://dx.doi.org/10.1590/S0066-

782X2008000200004

15. Rodrigues TC, Canani LH, Gross JL. Metabolic Syndrome, Insulin Resistance and Cardiovascular Disease in Type-1 Diabetes Mellitus.ArqBrasCardiol.2013;94(1):134139.DOI: http://dx.doi.org/10.1590/S0066782X2010000100020

16. Makluf ASD, Dias RC, Barra AA. Avaliação na qualidade de vida em mulheres com câncer de mama. Rev Bras Cancerol. 2006;52(1):49-58.

17.Zahar SEV, Aldrighi JM, Neto AMP, Conde DM, Zahar LO, Russomano F. Qualidade de vida em usuárias e não usuárias de terapia e reposição hormonal. Rev Asso Med Bras. 2004;51(3):133-138.2004.

18. Ribeiro JP, Rocha AS, Popim RC. Compreendendo o significado de qualidade de vida Segundo idosos portadores de diabetes mellitus tipo II. Esc Anna Nery. 2010;14(4):765-771.

19. Ciconelli RM, Ferraz MB, Santos W, Meinão I, Quaresma MR. Tradução para língua portuguesa e validação do questionário genérico de avaliação de qualidade de vida SF- 36(Brasil SF-36). Rev Bras de Reumatol. 1999;39(3).

20. Dias RS, Ramos CC, Kerr-Corrêa F, Trinca LA, Cerqueira ATAR, Dalben $l$, et al. Adaptação para o português do questionário de auto-avaliação de percepção de saúde física e mental da mulher de meia-idade Questionário da Saúde da Mulher. Rev. Psiq. Clín. 2002;29(4):181-189. 
21. Godoy-Matos AF, Oliveira J, Guedes EP, Carraro L, Lopes AC, Manfini MC, et al. Diretrizes brasileira de obesidade e da síndrome metabólica. $3^{\circ}$ Ed. Itapevi, SABESO; 2009.

22. Veiga EV, Nogueira MS, Cárnio EC, Marques S, Lavrador MAS, Moraes SA, et al. Avaliação de Técnicas da Medida da Pressão Arterial pelos Profissionais de Saúde. Arq Bras Cardiol. 2003;80(1):83-89. DOI: http://dx.doi.org/10.1590/S0066-

\section{$\underline{782 \times 2003000100008}$}

23. Rasia, J. A relação do sobrepeso e obesidade com desconfortos musculoesqueléticos de mulheres pósmenopausa. 2007;4(1):28-38.

24. Francischi $R$, et al. Obesidade: atualização sobre sua etiologia, morbidade e tratamento. Rev de Nutrição. 2000;13(1):1-21.

25. Oliveira AO, Mancini Filho J. Perfil nutricional e lipídico de mulheres na pósmenopausa com doença arterial coronariana. Arq Bras Cardiol. 2005;84(4).

26. Sociedade Brasileira de Cardiologia/ Sociedade Brasileira de Hipertensão/ Sociedade Brasileira de Nefrologia. VI Diretrizes Brasileiras de Hipertensão. Arq Bras Cardiol. 2010;95(1 supl.1):1-51.

27.Gomes GV, Vieira DR, Gomes AA, Maia NB, Correa APN, Freitas RF, et al. Comparação do autocuidado em mulheres pré e pós-menopausadas portadoras de Diabetes Mellitus Tipo II. 2014;06(01):53-59. DOI: 10.3895/S2175-08582014000100007

28. Galvão LLF, Farias MCS, Azevedo PRM, Vilar MJP, Azevedo GD. Prevalência de transtornos mentais comuns e avaliação da qualidade de vida no climatério. Ver Assoc Med Bras. 2007;53(5):414-420. DOI: http://dx.doi.org/10.1590/S010442302007000500017.
29. Lorenzi DRS, Baracat EC, Saciloto B, Padilha Jr I. Fatores associados à qualidade de vida na pós-menopausa. Rev Assoc Med Bras. 2006;52(5):312-314. DOI: http://dx.doi.org/10.1590/S010442302006000500017.

Recebido para publicação em 19/08/2014 Revisado em 02/09/2014 Aceito em 25/09/2014 